\title{
On generalizing generalized polygons
}

\author{
Andrew J. Woldar
}

\begin{abstract}
The purpose of this paper is to reveal in geometric terms a decade-old construction of certain families of graphs with nice extremal properties. Construction of the graphs in question is motivated by the way in which regular generalized polygons may be embedded in their Lie algebras, so that point-line incidence corresponds to the vanishing Lie product. The only caveat is that the generalized polygons are greatly limited in number. By performing successive truncations on an infinite root system of type $\widetilde{A}_{1}$, we are able to obtain an infinite series of incidence structures which approximate the behavior of generalized polygons. Indeed, the first two members of the series are exactly the affine parts of the generalized polygons of type $A_{2}$ and $B_{2}$.
\end{abstract}

Keywords: Turán problem, cage, large girth, generalized polygon, affine part, Lie algebra, root system

MSC 2000: 51E12, 05C35

\section{Introduction}

The material in this paper is based not on recent work but rather on undisclosed features of work that is now a decade old. At that time, our objective was to construct graphs which were extremal in the sense of the Turán problem for a fixed cycle of even length (see Section 2.1). The target asymptotic for this problem is provided by the upper bound which appears in the Even Circuit Theorem. However, this bound is known to be sharp in only a handful of cases, each corresponding to the existence of incidence graphs of regular generalized polygons. Such considerations led F. Lazebnik, V.A. Ustimenko and me to attempt to construct graphs which in some sense approximated the structure and behavior of these incidence graphs. Ultimately, our goal was realized with the construction of the doubly infinite family of graphs $\mathrm{CD}(k, q)$. 
Let $\mathcal{F}$ be a family of graphs, and let $\operatorname{ex}(v, \mathcal{F})$ be the greatest size $e$ of any graph of order $v$ which contains no subgraph isomorphic to a graph from $\mathcal{F}$. The Turán problem is to determine $\operatorname{ex}(v, \mathcal{F})$, or at least the asymptotic behavior of $\operatorname{ex}(v, \mathcal{F})(v \rightarrow \infty)$, for given $\mathcal{F}$. A more ambitious objective is to classify those such graphs which are extremal, i.e., of size $e=\operatorname{ex}(v, \mathcal{F})$.

Our present interest shall be in the case $\mathcal{F}=\left\{C_{2 t}\right\}$, where $C_{2 t}$ denotes the cycle of length $2 t$. In what follows, we shall write $\operatorname{ex}(v, 2 t)$ in place of the more cumbersome $\operatorname{ex}\left(v,\left\{C_{2 t}\right\}\right)$.

The best known upper bound in this case is given by the Even Circuit Theorem (see $[3,10]$ for proofs, although the result is almost universally attributed to Erdős). Specifically, the theorem states

$$
\operatorname{ex}(v, 2 t)=O\left(v^{1+\frac{1}{t}}\right)
$$

The Even Circuit Theorem has led many to speculate that

$$
\operatorname{ex}(v, 2 t)=\Omega\left(v^{1+\frac{1}{t}}\right)
$$

despite the fact that the only cases for which this bound is known to be sharp are $t \in\{2,3,5\}$. As previously mentioned, these are achieved by incidence graphs of regular generalized $m$-gons (where $m=t+1$ ).

As the next well known result shows, these "best performers" are greatly limited in number.

Theorem 2.1 (Feit-Higman [11]). Thick generalized $m$-gons exist only for $m \in$ $\{3,4,6,8\}$. Regular ones exist only for $m \in\{3,4,6\}$.

In fact, we conjecture that there are no other values of $t$ for which the bound in the Even Circuit Theorem is sharp. Thus, we regard these graphs as artifacts which falsely inflate one's perception of what is achievable in the Turán Problem when the family of forbidden subgraphs is limited to a single fixed cycle of even length.

\subsection{Related problems in extremal graph theory}

\subsubsection{Cage problem}

Fix integers $r \geq 2$ and $g \geq 3$. An $(r, g)$-graph is a regular graph of valency $r$ and girth $g$. An $(r, g)$-cage is an $(r, g)$-graph of minimum order. We denote the order of an $(r, g)$-cage by $v(r, g)$.

The cage problem can be simply stated as follows: For given $r$ and $g$, find all $(r, g)$-cages. A softening of this problem is to determine the orders of such cages, 



\subsubsection{Dense graphs of large girth}

By dense in this context, we mean "having many edges" with respect to a fixed girth. This is actually another formulation of the Turán Problem, where we choose the forbidden family $\mathcal{F}=\left\{C_{3}, C_{4}, \ldots, C_{g-1}\right\}$ to ensure that the girth be at least $g$.

Note that the goals here are the same as in previously stated Turán problem: to determine $\operatorname{ex}\left(v,\left\{C_{3}, \ldots, C_{g-1}\right\}\right)$ and, more ambitiously, to find all extremal graphs.

Interestingly, there is no current evidence to substantiate that the asymptotic behavior of the function $\operatorname{ex}\left(v,\left\{C_{3}, \ldots, C_{g-1}\right\}\right)$ differs in the slightest from that of ex $(v, g-2)$ provided $g$ is even. To clarify, this is not to say that the two corresponding "asymptotically equivalent" families of graphs coincide. Indeed, the Erdős-Rényi graphs [8] are extremal relative to avoiding a 4-cycle, however they contains 3-cycles. The point is that the number of edges in the Erdős-Rényi graphs are of the same asymptotic order as those of the (girth 6) generalized 3 -gons, though for the former graphs the constant is a bit smaller. (See $[15,18]$ for more general occurrences of this phenomenon.)

\subsubsection{Families of graphs with large girth}

For $i \geq 1$, let $G_{i}$ be a regular graph of valency $r$, girth $g_{i}$, and order $v_{i}$. Following Biggs [1], we say that $\left\{G_{i}\right\}$ is a family of graphs with large girth if

$$
g_{i} \geq \gamma \log _{r-1}\left(v_{i}\right)
$$

for some constant $\gamma$. It is well known that $\gamma \leq 2$ (e.g., see [2]), but no family has ever been found for which $\gamma=2$.

For many years the only significant results in this direction were the nonconstructive theorems of Erdős and Sachs with improvements by Sauer, Walther and others. These produced $\gamma=1$ (see [2, p. 107] for more details and references). The first explicit constructions were given by Margulis [21] with $\gamma \approx 0.44$ for some infinite families with arbitrarily large valency.

At present, the largest value that has been achieved is $\gamma=4 / 3$. These are realized by independent constructions of Margulis [21] and Lubotzky, Phillips, Sarnak $[20,25]$. Specifically, they are Cayley graphs of the group $\mathrm{PGL}_{2}\left(\mathbb{Z}_{q}\right)$ with respect to a set of $p+1$ generators, where $p, q$ are distinct primes congruent to $1 \bmod 4$ with the Legendre symbol $\left(\frac{p}{q}\right)=-1$.

Note that incidence graphs of regular generalized polygons fail to provide families with large girth because their girth never exceeds 12 . Still, we have introduced this extremal notion for a definite reason, see Section 3.2. 


\section{Graphs $\mathrm{CD}(k, q)$ and their properties}

The graphs $\mathrm{CD}(k, q)$ are the incidence graphs of the geometries we describe in this paper. As previously mentioned, the motivation behind their construction was to derive graphs which would have extremal properties approaching those of the incidence graphs of regular generalized polygons, yet be prolific in number. As it turns out, they are best described in terms of another family of graphs $\mathrm{D}(k, q)$ constructed in [14].

full screen

close

quit

\subsection{Constructions of graphs $\mathrm{D}(q), \mathrm{D}(k, q), \mathrm{CD}(k, q)$}

Let $q$ be a prime power, and let $P$ and $L$ be two copies of the countably infinite dimensional vector space $V$ over $\operatorname{GF}(q)$. Elements of $P$ will be called points and those of $L$ lines. In order to distinguish points from lines we introduce the use of parentheses and brackets: If $x \in V$, then $(x) \in P$ and $[x] \in L$. We adopt the following coordinate representations for points and lines:

$$
\begin{aligned}
(p) & =\left(p_{01}, p_{11}, p_{12}, p_{21}, p_{22}^{\prime}, p_{22}^{\prime \prime}, p_{23}, \ldots, p_{i i}^{\prime}, p_{i i}^{\prime \prime}, p_{i, i+1}, p_{i+1, i}, \ldots\right), \\
{[l] } & =\left[l_{10}, l_{11}, l_{12}, l_{21}, l_{22}^{\prime}, l_{22}^{\prime \prime}, l_{23}, \ldots, l_{i i}^{\prime}, l_{i i}^{\prime \prime}, l_{i, i+1}, l_{i+1, i}, \ldots\right) .
\end{aligned}
$$

We now define an incidence structure $(P, L, I)$ as follows. We say point $(p)$ is incident to line $[l]$ if the following coordinate relations hold:

$$
\begin{aligned}
p_{11}-l_{11} & =l_{10} p_{01} \\
p_{12}-l_{12} & =l_{11} p_{01} \\
p_{21}-l_{21} & =l_{10} p_{11} \\
& \vdots \\
p_{i i}^{\prime}-l_{i i}^{\prime} & =l_{10} p_{i-1, i} \\
p_{i i}^{\prime \prime}-l_{i i}^{\prime \prime} & =l_{i, i-1} p_{01} \\
p_{i, i+1}-l_{i, i+1} & =l_{i i}^{\prime} p_{01} \\
p_{i+1, i}-l_{i+1, i} & =l_{10} p_{i i}^{\prime \prime}
\end{aligned}
$$

We denote the incidence graph of $(P, L, I)$ by $\mathrm{D}(q)$.

Now, for each integer $k \geq 2$ we define the incidence structure $\left(P_{k}, L_{k}, I_{k}\right)$ as follows. First, $P_{k}$ and $L_{k}$ are obtained from $P$ and $L$, respectively, by projecting each vector (point or line) onto its initial $k$ coordinates. Incidence $I_{k}$ is then defined by imposing the first $k-1$ coordinate relations and ignoring all others. We denote by $\mathrm{D}(k, q)$ the incidence graph of the structure $\left(P_{k}, L_{k}, I_{k}\right)$. 
From an extremal point of view, the only discernable weakness of the graphs $\mathrm{D}(k, q)$ is that they become disconnected when $k \geq 6$, and continue to disconnect thereafter at regular intervals, see [17]. In every such case, however, the connected components of $\mathrm{D}(k, q)$ are pairwise isomorphic. We denote by $\mathrm{CD}(k, q)$ any one of these connected components.

Proofs of the following propositions may be found in [14] and [16], respectively.

Proposition 3.1. Let $q$ be any prime power, and $k \geq 2$. Then

(i) $\mathrm{D}(k, q)$ is a regular bipartite graph of valency $q$ and order $2 q^{k}$;

(ii) $\operatorname{Aut}(\mathrm{D}(k, q))$ is both vertex- and edge-transitive;

(iii) for even $k$, the girth of $\mathrm{D}(k, q)$ is at least $k+4$;

(iv) for odd $k$, the girth of $\mathrm{D}(k, q)$ is at least $k+5$.

Proposition 3.2. Let $\mathrm{CD}(k, q)$ denote a connected component of $\mathrm{D}(k, q)$. Then

(i) $\mathrm{CD}(k, q)$ is a regular, bipartite graph of valency $q$ and order $2 q^{k-\left\lfloor\frac{k+2}{4}\right\rfloor+1}$;

(ii) $\operatorname{Aut}(\mathrm{CD}(k, q))$ is both vertex- and edge-transitive;

(iii) for even $k$, the girth of $\mathrm{CD}(k, q)$ is at least $k+4$;

(iv) for odd $k$, the girth of $\mathrm{CD}(k, q)$ is at least $k+5$.

We believe the reader will concur that such manner of description belies any deep algebraic principles that may be involved in the construction of these graphs. Moreover, nondisclosure of such principles is in a sense excusable, since we are able to derive all important properties of these graphs directly from their coordinate relations.

\subsection{Graphs $\mathrm{CD}(k, q)$ as near-extremal structures}

We revisit the extremal problems described in Section 2, this time alluding to various improvements on asymptotics, courtesy of the graphs $\operatorname{CD}(k, q)$.

Regarding the two formulations of the Turán Problem, the best known general lower bound on each of $\operatorname{ex}(v, 2 t)$ and $\operatorname{ex}\left(v,\left\{C_{3}, C_{4}, \ldots, C_{2 t+1}\right\}\right)$ is provided, with one exception, by the graphs $\operatorname{CD}(k, q)$. Specifically, this bound is

$$
c_{t}\left(v^{1+\frac{2}{3 t-3+\epsilon}}\right)
$$

where $c_{t}$ is a positive constant and $\epsilon \in\{0,1\}$ depends on the parity of $t$. The excepted case, $t=5$, is achieved by the regular generalized hexagons. 


Let $\mathcal{L}=\mathcal{L}(G)$ denote the simple Lie algebra for $G=\mathrm{GL}(3, q)$ defined over the field $\operatorname{GF}(q)$. Recall that $\mathcal{L}$ is a nonassociative algebra with respect to the linear, skew-symmetric operation of Lie product, denoted $[$,$] , and that this operation$ satisfies the Jacobi identity: $[a,[b, c]]+[b,[c, a]]+[c,[a, b]]=0$.

Let $\Pi=\left\{r_{1}, r_{2}\right\}$ be a fundamental basis for $\mathcal{L}$. As usual, we refer to $r_{1}, r_{2}$ as the fundamental roots.

The Cartan matrix $A=\left(A_{i j}\right)$ of $\mathcal{L}$ is given by

$$
A=\left(\begin{array}{rr}
2 & -1 \\
-1 & 2
\end{array}\right) \text {. }
$$

From this matrix, one is able to generate the entire root system of $\mathcal{L}$ via linear extension of the action of the Weyl group $W=\left\langle w_{1}, w_{2}\right\rangle$ on the fundamental roots, specifically $r_{j}^{w_{i}}=r_{j}-A_{i j} r_{i}$. The resulting root system is given by $\Phi=$ $\Phi^{+} \cup \Phi^{-}$, where

$$
\Phi^{+}=\left\{r_{1}, r_{2}, r_{1}+r_{2}\right\} \quad \text { and } \quad \Phi^{-}=\left\{-r_{1},-r_{2},-\left(r_{1}+r_{2}\right)\right\} .
$$

We refer to the elements of $\Phi^{+}$as the positive roots of $\mathcal{L}$ with respect to $\Pi$, and to those of $\Phi^{-}$as the negative roots.

Now let $\Pi^{*}=\left\{r_{1}^{*}, r_{2}^{*}\right\}$ be the dual basis of $\Pi$, and define the contragredient action of $W$ on $\Pi^{*}$ via $r_{j}^{* w}(s)=r_{j}^{*}\left(s^{w^{-1}}\right)$. This gives us the dual root system

$$
\Phi^{*}=\left\{ \pm r_{1}^{*}, \pm r_{2}^{*}, \pm\left(r_{1}^{*}-r_{2}^{*}\right)\right\} .
$$

Recall the Cartan decomposition of a simple Lie algebra, namely

$$
\mathcal{L}=\mathcal{H} \oplus \mathcal{L}^{+} \oplus \mathcal{L}^{-}
$$

where $\mathcal{H}$ is the Cartan subalgebra of $\mathcal{L}$ (maximal self-normalizing subalgebra), and $\mathcal{L}^{+}$and $\mathcal{L}^{-}$are the positive and negative root spaces of $\mathcal{L}$, respectively. Subspaces $\mathcal{L}^{+}$and $\mathcal{L}^{-}$are a fortiori ideals of $\mathcal{L}$, but they are not subalgebras. However, the direct sums $\mathcal{L}^{U}=\mathcal{H} \oplus \mathcal{L}^{+}$and $\mathcal{L}^{L}=\mathcal{H} \oplus \mathcal{L}^{-}$are subalgebras of $\mathcal{L}$, which we call the upper and lower Borel subalgebras of $\mathcal{L}$, respectively. In what follows, we shall be mainly concerned with the upper Borel subalgebra $\mathcal{L}^{U}$.

Under the adjoint action of $\mathcal{H}$, each of $\mathcal{L}^{+}, \mathcal{L}^{-}$decomposes into a direct sum of $\mathcal{H}$-invariant subspaces, which we call root spaces. It is well known that every root space is one-dimensional. For each $s \in \Phi$, let $e_{s}$ be a corresponding (nonzero) root vector. In such manner, we get a further decomposition of $\mathcal{L}$ into the direct sum of $\mathcal{H}$ with the totality of root spaces. In particular, we see that $\left\{r_{1}^{*}, r_{2}^{*}, e_{r_{1}}, e_{r_{2}}, e_{r_{1}+r_{2}}\right\}$ is a basis for $\mathcal{L}^{U}$, presuming once more that $\mathcal{L}$ is the Lie algebra for $\mathrm{GL}(3, q)$. 
We are now ready to present our Lie algebraic model of $\mathrm{PG}(2, q)$. There are three types of points and three types of lines; in each case "type" is determined by the linear functional $s^{*} \in \Phi^{*}$ which is showing up as the lead term in each such expression.

Points: $\quad r_{1}^{*}$ (one element)

$-r_{1}^{*}+r_{2}^{*}+\lambda_{r_{1}} e_{r_{1}}$ (q elements)

$-r_{2}^{*}+\lambda_{r_{2}} e_{r_{2}}+\lambda_{r_{1}+r_{2}} e_{r_{1}+r_{2}}\left(q^{2}\right.$ elements)

Lines: $\quad r_{2}^{*}$ (one element)

$$
\begin{aligned}
& r_{1}^{*}-r_{2}^{*}+\lambda_{r_{2}} e_{r_{2}} \text { ( } q \text { elements) } \\
& -r_{1}^{*}+\lambda_{r_{1}} e_{r_{1}}+\lambda_{r_{1}+r_{2}} e_{r_{1}+r_{2}}\left(q^{2} \text { elements }\right)
\end{aligned}
$$

Incidence is defined by vanishing of the Lie product, i.e., point $p$ is incident to line $l$ if and only if $[p, l]=0$.

The reader will observe that each point and line is a specific linear combination relative to the basis $\left\{r_{1}^{*}, r_{2}^{*}, e_{r_{1}}, e_{r_{2}}, e_{r_{1}+r_{2}}\right\}$ of $\mathcal{L}^{U}$. However, most linear combinations have been excluded from consideration. This raises the question as to how such representative elements are chosen.

First, the lead term $s^{*} \in \Phi^{*}$ falls into one of two possible orbits under the action of the Weyl group $W$ on $\Phi^{*}$, namely $\left\{-r_{2}^{*}\right\}^{W}$ and $\left\{-r_{1}^{*}\right\}^{W}$. The choice here is a bit arbitrary, but we define points and lines in this respective order. Second, for a fixed $s^{*} \in \Phi^{*}$ an expression of the form $s^{*}+v$ is an admissible object (point or line) if and only if $s^{*}+v$ lies in the same orbit as $s^{*}$ under the action of the stabilizer $B$ in $\operatorname{GL}(3, q)$ of the flag $\left\{r_{1}^{*}, r_{2}^{*}\right\}$. From our explanation, the reader will note that there are precisely three orbits of points and three orbits of lines relative to this action (in each case, of respective sizes $1, q, q^{2}$ ), which is why we chose to list them separately and distinguish them by "type".

Observe that restriction of our model to the points $r_{1}^{*},-r_{1}^{*}+r_{2}^{*},-r_{2}^{*}$ and the lines $r_{2}^{*}, r_{1}^{*}-r_{2}^{*},-r_{1}^{*}$ yields the 6 -cycle, i.e., the thin generalized 3 -gon. However, it is a quite different substructure of $\mathrm{PG}(2, q)$ with which we will be concerned. We speak now of the affine part of PG $(2, q)$, which we define presently.

Fix a flag $\{p, l\}$ in $\mathrm{PG}(2, q)$. We define the affine part of $\mathrm{PG}(2, q)$ relative to $\{p, l\}$ to be the substructure induced on all points of maximum distance from $l$, and all lines of maximum distance from $p$. (In order to interpret "distance" more succinctly here, it may be convenient to consider the incidence graph of $\mathrm{PG}(2, q)$, where this notion corresponds to the usual one for graphs.) Because we are working inside a flag-transitive geometry, affine parts relative to different flags are isomorphic. 


\subsubsection{Lie algebraic model of the affine part of $\mathrm{PG}(2, q)$}

In terms of our Lie algebraic model of $\mathrm{PG}(2, q)$ given in 4.1.3, we may choose the affine part relative to the flag $\left\{r_{1}^{*}, r_{2}^{*}\right\}$. Then the points and lines at maximum distance from this flag correspond to the two largest orbits of $B$, each of size $q^{2}$. This gives us our desired model of the affine part:

Affine points: $\quad-r_{2}^{*}+\lambda_{r_{2}} e_{r_{2}}+\lambda_{r_{1}+r_{2}} e_{r_{1}+r_{2}}$

Affine lines: $\quad-r_{1}^{*}+\lambda_{r_{1}} e_{r_{1}}+\lambda_{r_{1}+r_{2}} e_{r_{1}+r_{2}}$

full screen

close

We hasten to point out that the affine part of $\operatorname{PG}(2, q)$ is not the affine plane $\operatorname{AG}(2, q)$, although both objects share the same point set. Whereas $\operatorname{AG}(2, q)$ is obtained by removing a single line from $\mathrm{PG}(2, q)$ ("line at infinity"), the affine part requires the removal of $q+1$ lines ("line at infinity" plus a parallel class). In particular, the incidence graph of this substructure is regular of valency $q$.

Example 4.1. We provide Lie algebraic models of the Fano plane PG $(2,2)$ and its affine part. In each case, lines are represented as subsets of points (the latter appear in boldface). The two structures are depicted in Figures 1 and 2, respectively.

Lines of the Fano plane:

$$
\begin{aligned}
& \left\{r_{1}^{*},-r_{2}^{*},-r_{2}^{*}+e_{r_{1}+r_{2}}\right\}=r_{1}^{*}-r_{2}^{*} \\
& \left\{r_{1}^{*},-r_{1}^{*}+r_{2}^{*}, r_{1}^{*}+r_{2}^{*}+e_{r_{1}}\right\}=r_{2}^{*} \\
& \left\{r_{1}^{*},-r_{2}^{*}+e_{r_{2}},-r_{2}^{*}+e_{r_{2}}+e_{r_{1}+r_{2}}\right\}=r_{1}^{*}-r_{2}^{*}+e_{r_{2}} \\
& \left\{-r_{2}^{*},-r_{1}^{*}+r_{2}^{*}+e_{r_{1}},-r_{2}^{*}+e_{r_{2}}+e_{r_{1}+r_{2}}\right\}=-r_{1}^{*}+e_{r_{1}} \\
& \left\{-r_{2}^{*}+e_{r_{2}},-r_{1}^{*}+r_{2}^{*}+e_{r_{1}},-r_{2}^{*}+e_{r_{1}+r_{2}}\right\}=-r_{1}^{*}+e_{r_{1}}+e_{r_{1}+r_{2}} \\
& \left\{-r_{1}^{*}+r_{2}^{*},-r_{2}^{*}+e_{r_{2}},-r_{2}^{*}+e_{r_{2}}+e_{r_{1}+r_{2}}\right\}=-r_{1}^{*}+e_{r_{1}+r_{2}} \\
& \left\{-r_{2}^{*},-r_{1}^{*}+r_{2}^{*},-r_{2}^{*}+e_{r_{2}}\right\}=-r_{1}^{*}
\end{aligned}
$$

Lines of the affine part of the Fano plane:

$$
\begin{aligned}
& \left\{-r_{2}^{*},-r_{1}^{*}+r_{2}^{*}+e_{r_{1}},-r_{2}^{*}+e_{r_{2}}+e_{r_{1}+r_{2}}\right\}=-r_{1}^{*}+e_{r_{1}} \\
& \left\{-r_{2}^{*}+e_{r_{2}},-r_{1}^{*}+r_{2}^{*}+e_{r_{1}},-r_{2}^{*}+e_{r_{1}+r_{2}}\right\}=-r_{1}^{*}+e_{r_{1}}+e_{r_{1}+r_{2}} \\
& \left\{-r_{1}^{*}+r_{2}^{*},-r_{2}^{*}+e_{r_{2}},-r_{2}^{*}+e_{r_{2}}+e_{r_{1}+r_{2}}\right\}=-r_{1}^{*}+e_{r_{1}+r_{2}} \\
& \left\{-r_{2}^{*},-r_{1}^{*}+r_{2}^{*},-r_{2}^{*}+e_{r_{2}}\right\}=-r_{1}^{*}
\end{aligned}
$$




\section{$1+0$ \\ construction is entirely general.}
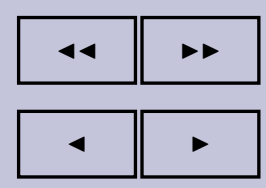

page 14 / 24

go back

full screen

close

quit

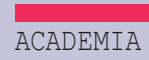

PRESS

WW

$\widehat{\underline{\underline{I I I I I}}}$

UNIVERSITEIT

GENT
There are seven types of rank two Chevalley groups, viz. $A_{2}, B_{2}, G_{2},{ }^{2} A_{3},{ }^{2} A_{4}$, ${ }^{3} D_{4},{ }^{2} F_{4}$. The first three are said to be of "normal type", the last four "twisted". Generalized 3-gons arise from $A_{2}$, generalized 4-gons from $B_{2},{ }^{2} A_{3}$ and ${ }^{2} A_{4}$, 6-gons from $G_{2}$ and ${ }^{3} D_{4}$, and 8-gons from ${ }^{2} F_{4}$. The types for which the generalized $m$-gons turn out to be regular are $A_{2}, B_{2}, G_{2}$ and ${ }^{2} A_{4}$. However, as the 4-gon coming from ${ }^{2} A_{4}$ defined over $\mathrm{GF}(q)$ is isomorphic to the one arising from $B_{2}$ defined over $\operatorname{GF}\left(q^{2}\right)$, it suffices to restrict our attention to $A_{2}, B_{2}$ and $G_{2}$.

Thus let $G$ be of type $A_{2}, B_{2}$ or $G_{2}$ defined over $\operatorname{GF}(q)$, where $q=p^{\alpha}$. Let $U$ be a fixed Sylow $p$-subgroup of $G$, and let $B$ be its normalizer in $G$. Then, as $G$ has rank two there are just two subgroups properly situated between $B$ and $G$. (In general, a rank $r$ group of Lie type has $2^{r}-2$ such intermediate subgroups, of which $r$ are maximal.) We denote these subgroups by $P_{1}, P_{2}$ and call them (maximal) parabolic subgroups of $G$. Groups $U$ and $B$ are commonly referred to as unipotent and Borel subgroups of $G$, respectively.

We now formulate our group geometric model for generalized $m$-gons as follows: Let $G$ be any rank two group of Lie type, and let $P_{1}, P_{2}$ be the parabolic subgroups of $G$ relative to a fixed choice of Borel subgroup $B$. We take as points all cosets of $P_{1}$ in $G$, and as lines all cosets of $P_{2}$ in $G$. We define point $x P_{1}$ to be incident to line $y P_{2}$ precisely when $x P_{1} \cap y P_{2} \neq \emptyset$.

Example 5.1. Let us specialize this setting to the one we have already seen in 4.1.2, where the rank two group is of type $A_{2}$, that is, $\mathrm{GL}(3, q)$. A convenient choice for Sylow $p$-subgroup here is the group of all upper triangular matrices with 1's along the diagonal. (This group has order $q^{3}$ so is surely a Sylow $p$-subgroup of $\mathrm{GL}(3, q)$.) In this case, $B$ is simply the group of all upper triangular matrices, which the reader will recall is the stabilizer of a flag. We may now identify $\operatorname{Stab}\left(V_{1}\right)$ and $\operatorname{Stab}\left(V_{2}\right)$ as the two parabolic subgroups $P_{1}, P_{2}$ of $\mathrm{GL}(3, q)$. Indeed, $\operatorname{Stab}\left(V_{1}\right)$ and $\operatorname{Stab}\left(V_{2}\right)$ are the only groups which lie strictly between $B$ and $\mathrm{GL}(3, q)$.

\subsection{Lie algebraic model}

This is a direct analogue of the Lie algebraic model of $\mathrm{PG}(2, q)$ presented in 4.1.3. Let $G$ be a rank two group of Lie type, and let $\mathcal{L}$ be its corresponding Lie algebra. We embed the points and lines of our generalized $m$-gon in the upper Borel subalgebra $\mathcal{L}^{U}$ as follows:

$$
\begin{array}{ll}
\text { Points: } & s^{*}+v, \text { where } s^{*} \in\left\{-r_{2}^{*}\right\}^{W} \text { and } s^{*}+v \in\left\{s^{*}\right\}^{B} \\
\text { Lines: } & s^{*}+v, \text { where } s^{*} \in\left\{-r_{1}^{*}\right\}^{W} \text { and } s^{*}+v \in\left\{s^{*}\right\}^{B}
\end{array}
$$

Incidence is once again defined in terms of the vanishing Lie product, cf. 4.1.3. 


\subsection{Lie algebraic model of affine part}

Appealing to 4.1.4, we define affine objects to be those at maximum distance from the flag $\left\{r_{1}^{*}, r_{2}^{*}\right\}$. Again, these objects comprise the two largest orbits under the action of the Borel subgroup $B$ of $G$, i.e., the stabilizer of the flag $\left\{r_{1}^{*}, r_{2}^{*}\right\}$. The resulting form of affine points is thus $-r_{2}^{*}+v$, and that of affine lines is $-r_{1}^{*}+v$, where in each case $s^{*}+v \in\left\{s^{*}\right\}^{B}$ for $s^{*} \in\left\{-r_{2}^{*},-r_{1}^{*}\right\}$.

Example 5.2. We provide Lie algebraic models for the generalized 4-gon of type $B_{2}$ and its affine part.

Points:

$$
\begin{aligned}
& r_{2}^{*} \text { (one element) } \\
& r_{1}^{*}-r_{2}^{*}+\lambda_{r_{2}} e_{r_{2}}(q \text { elements) } \\
& -r_{1}^{*}+r_{2}^{*}+\lambda_{r_{1}} e_{r_{1}}+\lambda_{2 r_{1}+r_{2}} e_{2 r_{1}+r_{2}}\left(q^{2}\right. \text { elements) } \\
& -r_{2}^{*}+\lambda_{r_{2}} e_{r_{2}}+\lambda_{r_{1}+r_{2}} e_{r_{1}+r_{2}}+\lambda_{2 r_{1}+r_{2}} e_{2 r_{1}+r_{2}}\left(q^{3}\right. \text { elements) }
\end{aligned}
$$

Lines: $\quad r_{1}^{*}$ (one element)

$$
\begin{aligned}
& -r_{1}^{*}+2 r_{2}^{*}+\lambda_{r_{1}} e_{r_{1}}(q \text { elements }) \\
& r_{1}^{*}-2 r_{2}^{*}+\lambda_{r_{2}} e_{r_{2}}+\lambda_{r_{1}+r_{2}} e_{r_{1}+r_{2}}\left(q^{2} \text { elements }\right) \\
& -r_{1}^{*}+\lambda_{r_{1}} e_{r_{1}}+\lambda_{r_{1}+r_{2}} e_{r_{1}+r_{2}}+\lambda_{2 r_{1}+r_{2}} e_{2 r_{1}+r_{2}}\left(q^{3} \text { elements }\right)
\end{aligned}
$$

Affine points: $\quad-r_{2}^{*}+\lambda_{r_{2}} e_{r_{2}}+\lambda_{r_{1}+r_{2}} e_{r_{1}+r_{2}}+\lambda_{2 r_{1}+r_{2}} e_{2 r_{1}+r_{2}}$

Affine lines: $\quad-r_{1}^{*}+\lambda_{r_{1}} e_{r_{1}}+\lambda_{r_{1}+r_{2}} e_{r_{1}+r_{2}}+\lambda_{2 r_{1}+r_{2}} e_{2 r_{1}+r_{2}}$

Note that our model for the generalized 4-gon involves four types of points and four types of lines. As alluded to above, these types correspond to Borel orbits, the two largest of which render the points and lines of the affine part of the 4-gon.

A similar model can be constructed for the generalized 6-gon of type $G_{2}$, however this exhausts all possibilities available to us. Our problem at this stage is more metaphysical than pragmatic: How to detect the essence of these structures so limited in number, and extrapolate on that essence. While we would like to claim some level of ingenuity in solving this problem, it turns out that the biggest role was played by the Beauty of Mathematics. It cried out to us where to look, and fortuitously, Ustimenko heard the message and interpreted it correctly.

\section{Affine Lie algebras}

Roughly speaking, the classification of simple Lie algebras runs parallel to that of Lie groups (over the complex numbers) and groups of Lie type (over finite 


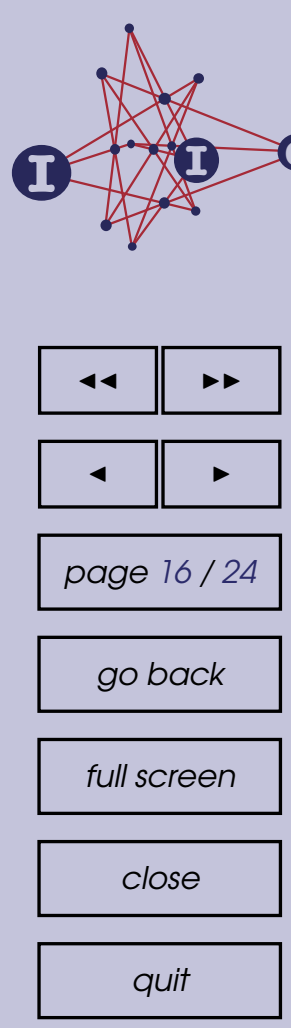

fields). There are some curious pathologies in the latter case, where a twisted group of Lie type will emerge which has no Lie group "relative", but such occurrences are rare and have been understood for a long time.

Less studied is the class of Kac-Moody algebras, though its subclass of affine Lie algebras has many important ties with Lie algebras. Both varieties give rise to (extended) Dynkin diagrams, (affine) Weyl groups and (affine) root systems, and may be constructed from their Cartan matrices.

Some differences, however, are striking. Whereas the root system of a Lie algebra is finite, that of an affine Lie algebra is infinite. This accounts for infinitely many root vectors, making affine Lie algebras infinite dimensional graded structures. Moreover, while all root spaces of a Lie algebra are one-dimensional, in the case of an affine Lie algebra this dimension may be larger.

The most crucial difference, however, is that for affine Lie algebras the connection to groups is far more tenuous. Still, such connections have been well studied and are attractively embodied in the general framework of "groups with a twin root datum (donnée radicielle)". This notion may be regarded as the group theoretical counterpart of the Kac-Moody algebra. We refer the reader to the lecture notes of P.-E. Caprace and B. Rémy [6] as an excellent introductory source, see also [5, 27].

\subsection{Affine Lie algebra of type $\widetilde{A}_{1}$}

In our preamble, we mentioned Dynkin diagrams. Every Lie algebra and affine Lie algebra has one. In the case of rank two Lie algebras of normal type, such diagrams consist of two nodes with some adjoining edges. The number of such edges conveys immediate information about the root system and Weyl group structure; ultimately it reveals all about the corresponding Lie algebra and Lie group. It is one of the most compact modes of description in all of mathematics.

In Figure 3, we give the Dynkin diagrams of all rank two Lie algebras of normal type. Recall that each such diagram is related to the existence of a regular generalized polygon.

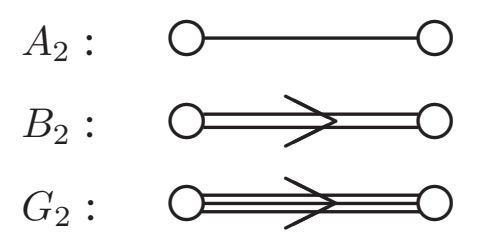

Figure 3: Dynkin diagrams of type $A_{2}, B_{2}, G_{2}$ 




Thus the Lie algebraic representation of $(p)$ is given by

$$
-r_{2}^{*}+p_{01} e_{r_{2}}+p_{11} e_{r_{1}+r_{2}}+p_{12} e_{r_{1}+2 r_{2}}+p_{21} e_{2 r_{1}+r_{2}}
$$

Let's now illustrate this same procedure for the line $[l]=\left[l_{10}, l_{11}, l_{12}, l_{21}\right]$. Here we have

$$
-r_{2}^{*}(\downarrow)[4]=\left\{e_{r_{1}}, e_{r_{1}+r_{2}}, e_{r_{1}+2 r_{2}}, e_{2 r_{1}+r_{2}}\right\} .
$$

The Lie algebraic representation for $[l]$ is thus given by

$$
-r_{1}^{*}+l_{10} e_{r_{1}}+l_{11} e_{r_{1}+r_{2}}+l_{12} e_{r_{1}+2 r_{2}}+l_{21} e_{2 r_{1}+r_{2}} .
$$

Subscripts of the form $\{i i\}$ which appear in such terms as $p_{i i}^{\prime}, p_{i i}^{\prime \prime}, l_{i i}^{\prime}, l_{i i}^{\prime \prime}$ are performing the exact same function. However, because the root space of each imaginary root $i r_{1}+i r_{2}$ is two-dimensional, we need two scalars for each $i \geq 2$ to fulfill this role. Thus $p_{i i}^{\prime}$ and $l_{i i}^{\prime}$ represent coefficients of $e_{i r_{1}+i r_{2}}^{\prime}$, and $p_{11}^{\prime \prime}$ and $l_{i i}^{\prime \prime}$ represent coefficients of $e_{i r_{1}+i r_{2}}^{\prime \prime}$, presuming of course that $\left\{e_{i r_{1}+i r_{2}}^{\prime}, e_{i r_{1}+i r_{2}}^{\prime \prime}\right\}$ is the chosen basis for the root space of $i r_{1}+i r_{2}$.

\subsection{Coordinate relations}

The first coordinate relation which appears in the definition of all graphs $\mathrm{D}(k, q)$ is

$$
p_{11}-l_{11}=l_{10} p_{01} .
$$

Our goal is to derive this relation, relying solely on our Lie algebraic model. Thus we may assume $k=2$, in which case point $(p)=\left(p_{01}, p_{11}\right)$ and line $[l]=\left[l_{10}, l_{11}\right]$ have respective representations given by

$$
\begin{gathered}
(p) \rightarrow X_{p}=-r_{2}^{*}+p_{01} e_{r_{2}}+p_{11} e_{r_{1}+r_{2}} ; \\
{[l] \rightarrow X_{l}=-r_{1}^{*}+l_{10} e_{r_{1}}+l_{11} e_{r_{1}+r_{2}} .}
\end{gathered}
$$

In terms of our model, $X_{p}$ and $X_{l}$ are incident if and only if $\left[X_{p}, X_{l}\right]=0$. So let's compute this Lie product in $\widetilde{\mathcal{L}}^{U}$, and see what happens:

$$
\begin{aligned}
{\left[X_{p}, X_{l}\right]=\left[-r_{2}^{*},\right.} & \left.-r_{1}^{*}\right]+\left[p_{01} e_{r_{2}},-r_{1}^{*}\right]+\left[p_{11} e_{r_{1}+r_{2}},-r_{1}^{*}\right]+\left[-r_{2}^{*}, l_{10} e_{r_{1}}\right] \\
& +\left[p_{01} e_{r_{2}}, l_{10} e_{r_{1}}\right]+\left[p_{11} e_{r_{1}+r_{2}}, l_{10} e_{r_{1}}\right]+\left[-r_{2}^{*}, l_{11} e_{r_{1}+r_{2}}\right] \\
& +\left[p_{01} e_{r_{2}}, l_{11} e_{r_{1}+r_{2}}\right]+\left[p_{11} e_{r_{1}+r_{2}}, l_{11} e_{r_{1}+r_{2}}\right] \\
=\left(p_{11}-\right. & \left.p_{01} l_{10}-l_{11}\right) e_{r_{1}+r_{2}}
\end{aligned}
$$

We conclude that $\left[X_{p}, X_{l}\right]=0$ if and only if $p_{11}-p_{01} l_{10}-l_{11}=0$, which is equivalent to the indicated coordinate relation. 
[11] W. Feit and G. Higman, The non-existence of certain generalized polygons, J. Algebra 1 (1964), 114-131.

[12] J. Hemmeter, V. A. Ustimenko and A. J. Woldar, Orbital schemes of $B_{3}(q)$ acting on 2-dimensional totally isotropic subspaces, European $J$. Combin. 19 (1998), 487-498.

[13] J. E. Humphreys, Introduction to Lie Algebras and Representation Theory, Springer, Berlin, 1994.

full screen

close

quit

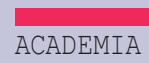

PRESS

WW

$\widehat{\underline{\text { IIIIII }}}$

UNIVERSITEIT

GENT

[14] F. Lazebnik and V. Ustimenko, Explicit construction of graphs with an arbitrary large girth and of large size, Appl. Discrete Math. 60 (1995), $275-284$.

[15] F. Lazebnik, V. A. Ustimenko and A. J. Woldar, Properties of certain families of 2k-cycle free graphs, J. Combin. Theory Ser. B 60 (1994), 293-298.

[16] _ A new series of dense graphs of high girth, Bull. Amer. Math. Soc. 32 (1995), 73-79.

[17] _ A characterization of the components of the graphs $\mathrm{D}(k, q)$, Discrete Math. 157 (1996), 271-283.

[18] _ Polarities and 2k-cycle free graphs, Discrete Math. 197/198 (1999), 503-513.

[19] F. Lazebnik and A. J. Woldar, General properties of some families of graphs defined by systems of equations, J. Graph Theory 38 (2001), $65-86$.

[20] A. Lubotzky, R. Phillips and P. Sarnak, Ramanujan graphs, Combinatorica 8 (1988), 261-277.

[21] G. A. Margulis, Explicit construction of graphs without short cycles and low density codes, Combinatorica 2 (1982), 71-78.

[22] M. Ronan, Lectures on Buildings, Academic Press, Boston, 1989.

[23] G. Royle, http://people.csse.uwa.edu.au/gordon/cages/allcages.html.

[24] H. Sachs, Regular graphs with given girth and restricted circuits, J. London Math. Soc. 38 (1963), 423-429.

[25] P. Sarnak, Some Applications of Modular Forms, Cambridge Univ. Press, Cambridge, 1990. 
